\title{
Zebrafish Olfactomedin 1 Regulates Retinal Axon Elongation In Vivo and Is a Modulator of Wnt Signaling Pathway
}

\author{
Naoki Nakaya, ${ }^{1}$ Hee-Sheung Lee, ${ }^{1}$ Yuichiro Takada, ${ }^{2}$ Itai Tzchori, ${ }^{3}$ and Stanislav I. Tomarev ${ }^{1}$ \\ ${ }^{1}$ Section of Molecular Mechanisms of Glaucoma, Laboratory of Molecular and Developmental Biology, National Eye Institute, ${ }^{2}$ Section for Translational \\ Research in Retinal and Macular Degeneration, National Institute on Deafness and Other Communication Disorders, and ${ }^{3}$ National Institute of Child Health \\ and Development, National Institutes of Health, Bethesda, Maryland 20892
}

Olfactomedin $1(\mathrm{Olfm} 1)$ is a secreted glycoprotein belonging to a family of olfactomedin domain-containing proteins. It is involved in the regulation of neural crest production in chicken and promotes neuronal differentiation in Xenopus. Here, we investigate the functions of Olfm1 in zebrafish eye development. Overexpression of full-length Olfm1, and especially its BMY form lacking the olfactomedin domain, increased the thickness of the optic nerve and produced a more extended projection field in the optic tectum compared with control embryos. In contrast, injection of olfm1-morpholino oligonucleotide (0lfm1-M0) reduced the eye size, inhibited optic nerve extension, and increased the number of apoptotic cells in the retinal ganglion cell and inner nuclear layers. Overexpression of full-length Olfm1 increased the lateral separation of the expression domains of eye-field markers, $r x 3$ and six3. The 0lfm1-M0 had the opposite effect. These data suggest that zebrafish 0lfm1 may play roles in the early eye determination, differentiation, optic nerve extension, and branching of the retinal ganglion cell axon terminals, with the N-terminal region of Olfm1 being critical for these effects. Injection of RNA encoding WIF-1, a secreted inhibitor of Wnt signaling, caused changes in the expression pattern of $r x 3$ similar to those observed after Olfm1-MO injection. Simultaneous overexpression of WIF-1 and 0lfm1 abolished the WIF-1 effect. Physical interaction of WIF-1 and Olfm 1 was demonstrated by coimmunoprecipitation experiments. We concluded that Olfm1 serves as a modulator of Wnt signaling.

Key words: eye development; axonal extension; neuronal differentiation; Wnt signaling; WIF-1; morpholino oligonucleotides

\section{Introduction}

Olfactomedin 1 (Olfm1), also known as noelin in chicken and Xenopus, and as pancortin in mouse and rat, is a secreted glycoprotein that was originally identified in the bullfrog olfactory neuroepithelium (Snyder et al., 1991; Nagano et al., 1998; Barembaum et al., 2000). A conserved domain in the C-terminal part of Olfm1 was named the olfactomedin domain and is found in a family of proteins consisting of at least 13 members in mammals (Zeng et al., 2005). Some family members, such as latrophilins and gliomedin, are membrane-bound proteins containing the olfactomedin domain in the extracellular $\mathrm{N}$-terminal regions, whereas the intracellular C-terminal domains of these proteins are essential for the transduction of extracellular signals to intracellular signaling pathways (Volynski et al., 2004; Eshed et al., 2005). Other family members, similar to Olfm 1 , are secreted glycoproteins whose functions are mostly unknown. Mutations in

Received Feb. 11, 2008; revised May 23, 2008; accepted June 18, 2008.

This work was supported by the National Eye Institute intramural research program. We thank David Rawnsley for his participation in some experiments during his summer student training program at the National Eye Institute. We thank Drs. Heiner Westphal, Igor Dawid, and Reiko Toyama for their suggestions and Drs. Olof Sundin and Janine Davis for suggestions and critical reading of this manuscript. We thank Dr. Hiroko Kataoka for providing runx constructs, Drs. Aiwu Cheng and Mark Mattson for providing eyes from Olfm 1 null mice, Drs. J. Nathans and J.C. Hsieh for providing WIF-1-lgG and WD-IgG fusion constructs, Dr. R. Fariss for help with the confocal microscope, and Dr. R. Moon for providing the Super8XTOPFlash reporter construct.

Correspondence should be addressed to Stanislav Tomarev, National Eye Institute, National Institutes of Health, Building 7, Room 103, 7 Memorial Drive, MSC 0704, Bethesda, MD 20892-0704. E-mail: tomarevs@nei.nih.gov.

D01:10.1523/JNEUROSCI.0617-08.2008

Copyright $\odot 2008$ Society for Neuroscience $\quad$ 0270-6474/08/287900-11\$15.00/0 one of the secreted olfactomedin-domain containing proteins, myocilin, contribute to open-angle glaucoma (Stone et al., 1997), an eye disease characterized by the death of retinal ganglion cells (RGCs), degeneration of axons in the optic nerve, and a specific deformation of the optic nerve head. Most glaucoma-causing mutations in myocilin are located in the olfactomedin domain and lead to the accumulation of the mutated protein inside the cells with subsequent apoptotic cell death (Joe et al., 2003; Liu and Vollrath, 2004).

In Xenopus, olfm 1 mRNA was detected mainly in postmitotic neurogenic tissues in the developing central and peripheral nervous systems, first appearing after neural tube closure (Moreno and Bronner-Fraser, 2001, 2002, 2005). In chicken, Olfm1 is involved in generation of the neural crest cells (Barembaum et al., 2000). Four structurally different mRNAs, named $A M Y, B M Y$, $A M Z$, and $B M Z$, are produced from the Olfml gene (Danielson et al., 1994). They share a common central region (M) and have two different $5^{\prime}$-regions (A or B) transcribed from distinct promoters, and two different $3^{\prime}$-regions ( $\mathrm{Y}$ and $\mathrm{Z}$ ) produced by alternative splicing (Danielson et al., 1994). The AMY and BMY forms encode a shorter form of Olfm 1 lacking the olfactomedin domain that is encoded by two exons found in the $A M Z$ and $B M Z$ forms.

The zebrafish genome contains two olfm 1 genes, olfm $1 a$ and olfm $1 \mathrm{~b}$, located on chromosomes 5 and 21, respectively. At least four transcripts are produced from each olfm 1 gene (Nakaya and Tomarev, 2007). The distributions of different transcripts of olfm1 genes during embryonic development [16-55 h postfertil- 
ization (hpf)] have been reported previously in detail (Nakaya and Tomarev, 2007).

In this study, we investigate possible roles of the olfml genes in zebrafish eye development using gene silencing by morpholino oligonucleotides (MOs) and overexpression of different Olfm1 isoforms. We demonstrate that suppression of Olfm1 expression leads to multiple developmental defects, including reduction of eye size and eye pigmentation, retinal degeneration, and inhibition of the optic nerve growth. In contrast, the overexpression of Olfm1, and especially Olfm 1 variants lacking the olfactomedin domain, produces a thicker optic nerve with an extended terminal area in the optic tectum. We show that Olfm 1 physically interacts with WIF-1, a secreted inhibitor of Wnt signaling. Olfm 1 and WIF-1 have antagonistic effects, and their interaction may fine tune Wnt activity.

\section{Materials and Methods}

Husbandry of fishes. Wild-type zebrafish were maintained as described by Westerfield (2000). Embryos were produced by natural matings. For some experiments, pigmentation of embryos was reduced by adding 0.2 mM 1-phenyl-2-thiourea to water. All experiments using animals were approved by the National Eye Institute Animal Use and Care Committee.

Frozen sections and immunofluorescence. Zebrafish embryos were dechorionated and fixed with $4 \%$ paraformaldehyde in $0.1 \mathrm{M} \mathrm{PBS,} \mathrm{pH} \mathrm{7.4,} \mathrm{at}$ $4^{\circ} \mathrm{C}$ overnight. For frozen sectioning, fixed embryos were equilibrated in $30 \%$ sucrose and embedded into OCT Compound (Electron Microscopy Sciences). Frozen sections $(5 \mu \mathrm{m})$ were produced using a cryostat (CM3050; Leica). Frozen sections were blocked in 5\% normal goat serum and then incubated with anti-Olfm 1 antibody (1:500 dilution), anti-HuC/D (1:400 dilution; Abcam), Zn-5 (1:500; Oregon Monoclonal Bank), anti-PKC $\beta 1$ (1:300 dilution; Santa Cruz Biotechnology), or Zpr-1 (1:200 dilution; Zebrafish International Resource Center). Slides were washed in PBS and then incubated with Alexa488- or Alexa595-conjugated goat anti-mouse IgG (1:400 dilution; Invitrogen) and with $4^{\prime}, 6^{\prime}$-diamidino2-phenylindole (DAPI) for nuclear staining. Images were collected using a confocal laser microscope (TCS SP2; Leica) or an Axoplan2 microscope (Carl Zeiss).

In situ RNA hybridization. Zebrafish olfm1 TR2 and TR4-specific probes were produced as described previously (Nakaya and Tomarev, 2007). TrkC1, six3, and $r \times 3$ cDNAs were purchased from Open Biosystems. Digoxigenin (DIG)-labeled cRNA probes were synthesized by in vitro transcription of the corresponding linearized plasmids using SP6, T7, or T3 polymerases and DIG-RNA labeling mixture (Roche). Wholemount in situ hybridization was performed as described previously (Toyama et al., 1995). After visualization of in situ hybridization signals, some embryos were embedded in OCT, and $10 \mu \mathrm{m}$ frozen sections were prepared. The mounted sections were observed under an Axoplan2 microscope.

Olfm1 antibody production. Mouse anti-Olfm 1 antibody was produced against the KFKQVEESHKQHLARQ peptide using the Custom Antibody Production Service of the University of Virginia. Specificity of the antibody was validated by Western blot experiments with two types of cell lysates. First, lysates of COS-7 cells and COS-7 cells transfected with mouse Olfm1, mouse optimedin, and mouse myocilin were used. Second, eye extracts were prepared from wild-type mice and null mice that lacked exons 2 and 3 encoding the M part of Olfm1 (Cheng et al., 2007).

Western blot. Whole embryos were homogenized in a lysis buffer (10 mм Tris-HCl, pH 7.5, 1 mм EDTA, 150 mm NaCl, 1\% Triton X-100, 5 $\mathrm{mm} \mathrm{NaF}, 0.5 \mathrm{~mm}$ sodium orthovanadate, $10 \%$ glycerol, $1 \mathrm{~mm}$ dithiothreitol, $1 \mathrm{~mm}$ PMSF, $1 \mu \mathrm{g} / \mathrm{ml}$ aprotinin, $1 \mu \mathrm{g} / \mathrm{ml}$ leupeptin, and $1 \mu \mathrm{g} / \mathrm{ml}$ pepstatin) for $20 \mathrm{~min}$ on ice. After centrifugation, the soluble fraction was collected and protein concentration was determined using the BCA assay kit (Pierce). The extracted proteins $(15 \mu \mathrm{g})$ were separated on an $12 \%$ SDS-PAGE gel and transferred to a polyvinylidene difluoride membrane (Invitrogen). A membrane was incubated with anti-Olfm1 antibody (1:10 dilution) followed by anti-mouse IgG antibody conjugated to horseradish peroxidase (HRP) (1:5000 dilution; GE Healthcare). The

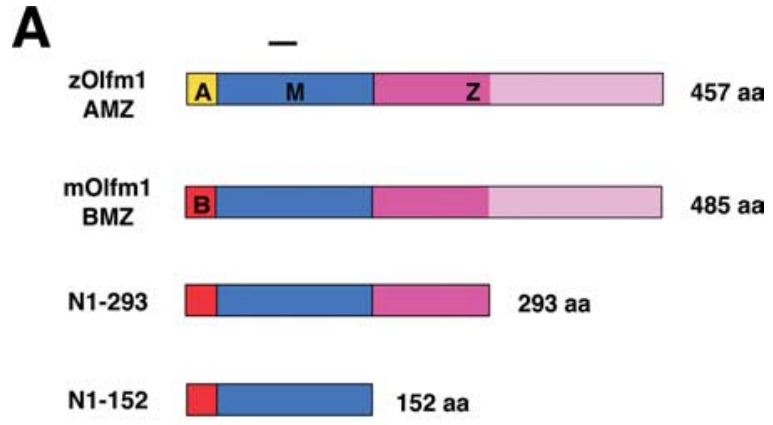

B
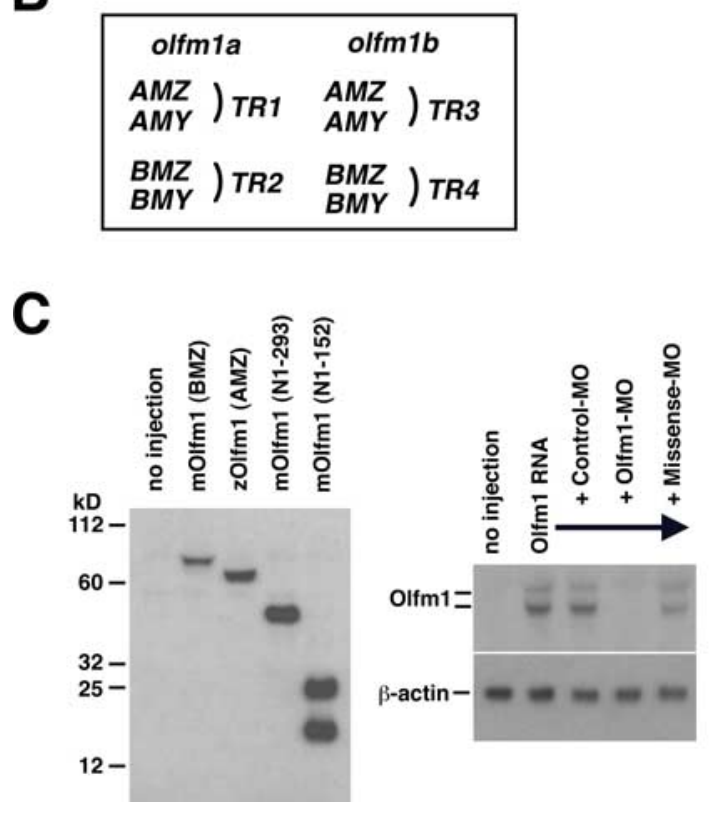

Figure 1. A, Schematic diagram of the 0 lfm 1 constructs used in this study. $A, M$, and $Z$ denote different protein regions. The A or B regions (shown in yellow or red, respectively) contain a signal peptide, the $M$ region (shown in blue) is common between different $01 \mathrm{fm} 1$ forms, and the $Z$ region (shown in dark and light pink) contains the olfactomedin domain (shown in light pink). $\boldsymbol{B}$, The nomenclature of transcripts of zebrafish olfm $1 a$ and olfm 16 genes. C, Expression of different $0 \mathrm{lfm} 1$ forms $24 \mathrm{~h}$ after injection of $0.1 \mathrm{ng}$ of corresponding RNAs at cell stage 1. Total soluble proteins were analyzed by Western blotting using the monoclonal antibody against the peptide in the $\mathrm{M}$ domain (left). The right demonstrates inhibition of 0 lfm 1 by Olfm1-M0 coinjection. Mouse Olfm1 RNA preceded by the zebrafish sequence sensitive to Olfm1-M0 was synthesized and injected into cell soma of cell stage 1 embryos with or without an injection of the 0 lfm1-M0, control M0, and missense $\mathrm{M} 0$ into the egg yolk. The Olfm1-M0 completely inhibited the 0lfm1 protein expression (appeared as upper glycosylated and lower unglycosylated bands) from the injected RNA in the bud stage embryos.

HRP signals were detected using a chemiluminescence detection kit (SuperSignal Femto Dura Extended Duration Substrate; Pierce) and a BioMax MS film (Eastman Kodak).

Whole-mount immunofluorescence. Anti-acetylated tubulin antibody was purchased from Sigma-Aldrich. Alexa 488-conjugated secondary antibody was purchased from Invitrogen. Zebrafish embryos were dechorionated and fixed with $4 \%$ paraformaldehydes in PBS at $4^{\circ} \mathrm{C}$ overnight. Embryos were incubated in blocking buffer ( $1 \%$ bovine serum albumin, $0.1 \%$ Triton X-100, $0.05 \%$ dimethylsulfoxide, and $2 \%$ goat serum in PBS) at $4^{\circ} \mathrm{C}$ for $1 \mathrm{~h}$ and then at $4^{\circ} \mathrm{C}$ overnight in blocking buffer containing the primary antibody. Embryos were washed three times with PBS-T (0.1\% Triton X-100 in PBS) for $1 \mathrm{~h}$ and then incubated with the fluorescence-labeled secondary antibody at $4^{\circ} \mathrm{C}$ overnight. Embryos were washed three times with PBS-T for $1 \mathrm{~h}$ and mounted on a glass slide in $0.5 \%$ glycerol. Images were collected using a Carl Zeiss Axoplan2 
fluorescent microscope equipped with a deconvolution software (Axovision; Carl Zeiss).

Terminal deoxynucleotidyl transferasemediated biotinylated UTP nick end labeling staining. Zebrafish frozen sections $(6 \mu \mathrm{m})$ were incubated with rabbit anti-ssDNA antibody (IBL USA) at $4^{\circ} \mathrm{C}$ overnight. After washing in PBS, sections were incubated with Alexa 488 anti-rabbit IgG/DAPI. Immunofluorescence images were collected with a Leica TCS SP2 confocal laser microscope.

Morpholino and RNA injections. The $\mathrm{MO}$ antisense oligonucleotide to knockdown Olfm 1 expression in embryos was designed and produced by Gene Tools. The sequences of oligonucleotides used in these experiments were as follows: control MO, 5'-CCTCTTACCTCAGTTACAATTTATA-3'; Olfm1-MO, 5' AGCAAAGGCACCGACATCTCTGCTC-3'; missense MO, 5' -AGGAAACGCACCCACATGTCTCCTC-3'.

The MOs were dissolved in distilled water, mixed with $2 \times$ injection buffer $(0.05 \%$ phenol red, $240 \mathrm{~mm} \mathrm{KCl}$, and $40 \mathrm{~mm}$ HEPES, pH 7.4), and injected into egg yolk of cell stage 1-4 embryos by a Pneumatic PicoPump (PV820; World Precision Instruments). For rescue experiments, the insert of cDNA clone CO250150 encoding the full-length AMZ form of zebrafish Olfm $1 \mathrm{~b}$ obtained from Open Biosystems was subcloned into the pCS2+ vector. Mouse Olfm1 deletion constructs, N1-293 and N1-152, were prepared by PCR amplifications from the full-length mouse Olfm 1 cDNA clone BC026547 using the following primers: ataagcttgccgccaccatgtcggtgccgctgctgaa [forward (FW)], attctagaatgtcggtgccgctgctgaa [N1293 reverse (RV)], and attctagaagcctacttgaactgcctggctagat (N1-152-RV). Zebrafish wif-1 was amplified by PCR from cDNA clone BC091959 (Open Biosystems) and cloned into the pCS2 + vector. Identity of all selected clones was confirmed by sequencing. RNA was synthesized using a mMESSAGE mMACHINE kit (Ambion) and different pCS2 constructs as templates. Synthesized RNAs $(0.02-0.1 \mathrm{ng})$ were injected into one-cell stage embryos with/without the indicated MOs.

Quantitative reverse transcription-PCR. Ten embryos from each developmental stage were dechorionated and homogenized in $500 \mu \mathrm{l}$ of Trizol (Invitrogen) using a Polytron (PT-MR2100; Kinematica) at the maximum power for $1 \mathrm{~min}$. Total RNA was isolated from the lysates following the protocol of the manufacturer. RNA was dissolved in distilled water at concentrations of $0.1-0.3 \mu \mathrm{g} / \mu \mathrm{l}$. Quantitative (Q) PCR was performed using $0.5 \mu \mathrm{g}$ of total RNA as described previously (Hemish et al., 2003). Briefly, RNA was converted to cDNA using Taqman Reverse Transcription Reagents (Applied Biosystems) and then dispensed into a 96-well PCR plate. Forward and reverse primers for a target gene and SYBR Green PCR Master Mix (Applied Biosystems) were added into each wells, and PCR reaction was performed and monitored using a 7900HT Real Time Thermocycler (Applied Biosystems). Elongation factor $1 \alpha(E F 1 \alpha)$ was used for normalization after a confirmation that its expression was not dramatically changed during developmental stages under study. Primers used for the Q-PCR were as follows: efl $\alpha$ (FW), 5'-CAGTGCTGGATTGCCACACT-3'; efl $\alpha$ (RV), 5'-CCAGAACGACGGTCGATCTT-3'; TR2 (FW), 5' -CGATGATCACCAACTGGATGTC-3'; TR2 (RV), 5'-GCCACGCTTAATTTGGTGCTA-3'; TR4 (FW), 5'-GGTGCTCAGCACCATGGC-3'; TR4 (RV), 5'-GCGGTCAGTTTGGTGGTGTT-3'; Olfmla Y form (FW), 5'-GCTGCAGAGACTGAAGAACAAGTT-3'; Olfmla Y form (RV), 5'-AACTGAATGATGCGATCAGTTGAC-3'; Olfmla Z form (FW), 5'-CCACAACAGGGTGTCTAATCTTGA3'; Olfm $1 a$ Z form (RV), 5' -AGTCAGCTTCCCACAGGCTAAT-3'; Olfm $1 b$ Y form (FW), 5'-GAGAGCCAGAGGCCAGAAGAA-3'; Olfm $1 b$ Y form (RV), 5'-CGTGACATAAACAGCGTGACTGA-3'; Olfm $1 b$ Z form (FW), 5'-TGCAGAATCTGACTGCGAGTCT-3'; Olfm $1 b$ Z form (RV), 5'-GGAGTGCAGGTCATCATAGTCATAA-3'.
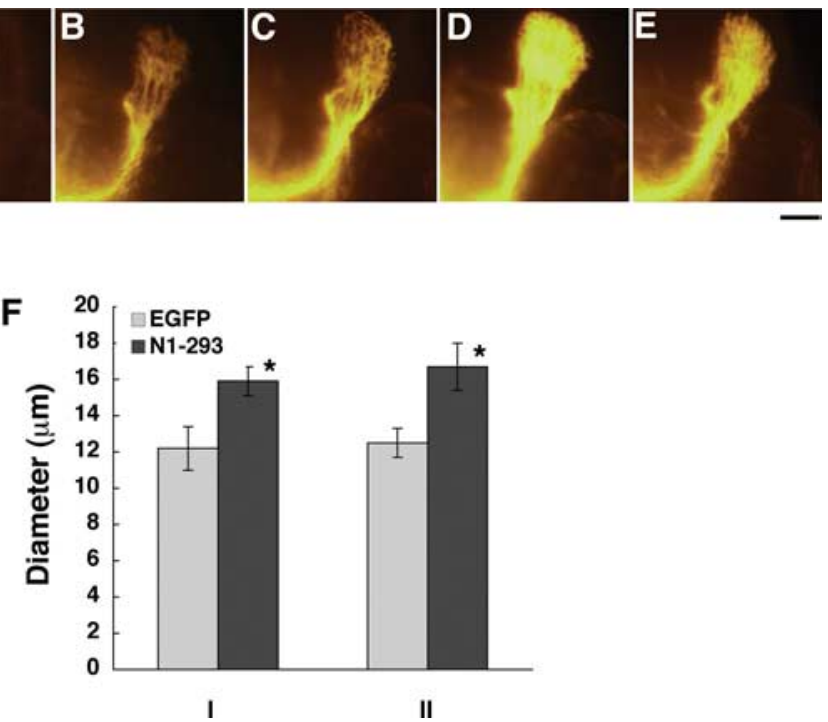

II erase plasmid (phRL-SV40, 2.5 pg; Promega) were injected to one-cell stage embryos together with or without enhanced green fluorescent protein (EGFP), wif-1, and/or olfm1 RNAs (25 pg). The luciferase activities in 24 hpf embryos were determined using a Luciferase Assay kit (Promega) as in the protocol of the manufacturer. Briefly, 10 embryos were homogenized in the lysis buffer $(100 \mu \mathrm{l})$, and the supernatant was collected after spinning at $15,000 \times g$ for $15 \mathrm{~min}$. The firefly and renilla luciferase activities in the supernatant were sequentially determined by adding the Luciferase Assay Substrate and the STOP\&GROW solutions and using a multiplate luminometer (VICTOR-light; PerkinElmer Life and Analytical Sciences).

Optic nerve analysis. DiI injection was performed using the previously described method (Yoda et al., 2004). Briefly, embryos were fixed with $4 \%$ paraformaldehyde and placed in $1.2 \%$ low melting agarose. DiI $(0.5$ $\mathrm{nl}, 75 \mathrm{mg} / \mathrm{ml}$ ) was injected into two positions diagonal to each other (nasodorsal and temporovental) in the left eye. The embryos were incubated at $28^{\circ} \mathrm{C}$ overnight, and the projection of the optic nerve stained with DiI was observed and photographed using a dissection fluorescence microscope (Stemi SV11; Carl Zeiss). The diameter of the optic nerves was measured and calculated using the NIH Image software.

Coimmunoprecipitation. HEK-293 cells were grown in $60 \mathrm{~mm}$ plates and transfected with Flag-tagged Olfm1 (Torrado et al., 2002) and WIF-1 tagged with the human heavy chain IgG (Hsieh et al., 1999) cDNA constructs using Lipofectamine 2000 (Invitrogen). (WIF-1 constructs were kindly provided by Dr. Jen-Chih Hsieh, State University of New York at Stony Brook, Stony Brook, NY). At $2 \mathrm{~d}$ after transfection, conditioned medium was collected and centrifuged at $15,000 \times g$ for $15 \min$ at $4^{\circ} \mathrm{C}$. To reduce background, conditioned medium was incubated with proteinG agarose (Roche) beads for $1 \mathrm{~h}$ at $4^{\circ} \mathrm{C}$ in a rotary shaker. The beads were removed by centrifugation, and the monoclonal Flag antibody beads were added to precleared conditioned medium for $2 \mathrm{~h}$ at $4^{\circ} \mathrm{C}$ in a rotary shaker. The beads were precipitated and washed three times with lysis buffer. Bound proteins were eluted by a 3XFLAG peptide (Sigma) and analyzed by Western blotting as described above. The peroxidaseconjugated antibodies against human IgG (1:1000 dilution) were used for detection of WIF-1 protein.

Statistical analysis. Data were analyzed using the unpaired Student's $t$ test preceded by $F$ test for variances. 

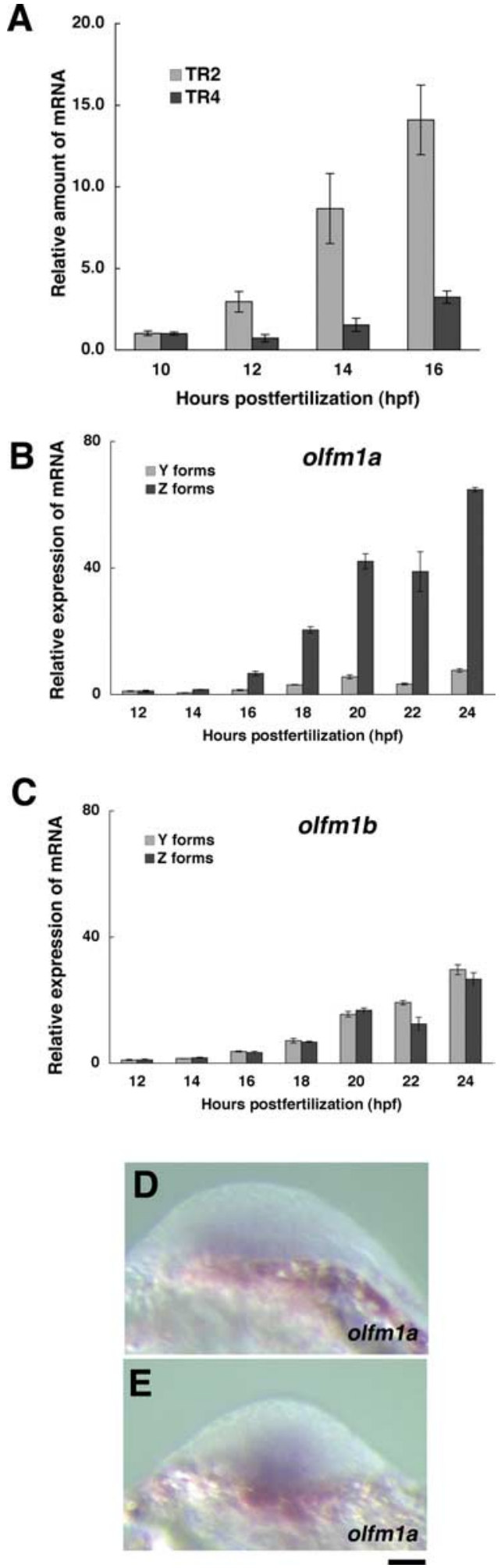

Figure 3. Expression of olfm $1 \mathrm{mRNAs}$ in the developing zebrafish embryos. $\boldsymbol{A}$, Expression kinetics of olfm $1 a$ (the $T R 2$ form corresponding to $B M Z$ and $B M Y$ transcripts) and olfm $1 b$ mRNAs (the TR4 form corresponding to BMZ and BMY transcripts) in the course of early development. Total RNA was isolated from 10 embryos for each time point. Primers specific for different groups of transcripts were used for Q-PCR analysis. The amplified DNA fragments produced single bands as judged by agarose gel electrophoresis. Values on the $y$-axis represent the amount of mRNA for each time point shown relative to that of $10 \mathrm{hpf}$ embryos. The average amounts of amplification cycles were 28.6 and 32.3 for TR2 and TR4, respectively, for $16 \mathrm{hpf}$ embryos. Normalization was performed relative to the EF1a gene expression. $B, C$, Expression kinetics of shorter $(\mathrm{Y})$ and longer ( $\mathrm{Z}$ ) forms of olfm $1 \mathrm{mRNAs}$ in the developing zebrafish embryos. Values on the $y$-axis represent the amount of mRNA for each time point shown relative to

\section{Results}

Overexpression of Olfml increases thickness of the retina and the optic nerve

Olfm1 is a conserved protein showing a $85 \%$ identity of amino acid sequence between zebrafish and mouse (Nakaya and Tomarev, 2007). To test effects of overexpression of different forms of Olfm1, mRNAs encoding the full-length AMZ form of zebrafish Olfm1 (zOlfm1b) and truncated forms of mouse Olfm1 (Fig. 1A) were injected into zebrafish embryos. N1-293 mRNA encodes the BMZ form of mouse Olfm1 without olfactomedin domain, whereas N1-152 mRNA encoded the BMY form of mouse Olfm 1 . The nomenclature of transcripts of zebrafish olfm 1 genes is shown in Figure $1 \mathrm{~B}$. Injection of RNAs encoding $\mathrm{zOlfm} 1$ or N1-293 into embryos induced synthesis of a protein migrating as a single band with apparent molecular weight of 70 or $50 \mathrm{kDa}$, respectively (Fig. 1C, left). These apparent molecular weights were higher than the predicted molecular weights of 52.5 and 33.4 $\mathrm{kDa}$, respectively, indicating carbohydrate modification of proteins encoded by these constructs. The levels of endogenous Olfm 1 are low at this stage of development, and Olfm1 bands were masked by the background bands. RNA encoding the N1152 form produced two protein bands on the gel, with apparent molecular weights of 24.6 and $15.9 \mathrm{kDa}$, most probably corresponding to modified and unmodified proteins, respectively. Because N1-152 is contained within the N1-293 protein, this indicates that the N1-152 protein is not modified as efficiently as longer proteins containing full or partial $\mathrm{Z}$ region. When expression constructs were transfected into HEK-293 cells, proteins with apparent molecular weights that were higher than calculated molecular weights were also produced. Treatment of cell lysates with a mixture of glycosidases [PNGaseF, $\beta-N$ acetylglucosaminidase, $\beta$-1-4-galactosidase, $o$-glycosidase and $\alpha(2-3,6,8,9)$ neuraminidase] reduced the apparent molecular weights of different Olfm 1 forms to predicted molecular weights, confirming that modification of Olfm1 is mainly attributable to glycosylation (data not shown).

Injection of $20 \mathrm{pg}$ of RNAs encoding different forms of zebrafish or mouse Olfm 1 did not induce obvious morphological changes. The general structure of the eye or retina did not change significantly after zOlfm 1 RNA injection in 72 hpf embryos (data not shown). However, the full-length zOlfm1, but not truncated mouse forms, produced a statistically significant increase in the thickness of the inner nuclear layer and the total retina compared with EGFP injected embryos (69.12 \pm 2.37 vs $60.07 \pm 0.56 \mu \mathrm{m}$, $p<0.05$ and $150.04 \pm 3.73$ vs $132.27 \pm 3.30 \mu \mathrm{m}, p<0.01$, respectively).

To analyze changes in the optic nerve after overexpression of Olfm1, DiI was injected into the eyes of $72 \mathrm{hpf}$ embryos, and the thickness and the arborization of the optic nerve was analyzed $16 \mathrm{~h}$ later. Injection of zOlfm 1 and especially truncated N1-152 and N1-293 constructs increased the thickness of the optic nerve and produced a broader projection field in the optic tectum compared with control embryos (Fig. 2A-E). Measurements of the optic nerve at two positions (exit from the optic globe and optic chiasm level) demonstrated its increased thickness after injection

$\leftarrow$

that of $12 \mathrm{hpf}$ embryos. The average amounts of amplification cycles were 30.8, 28.6, 32.0, 32.3, 29.3, 27.5, 29.8, and 30.6 for TR1-TR4, olfm $1 a \mathrm{Y}$ and $Z$ forms, and olfm $1 b Y$ and $Z$ forms, respectively, for $16 \mathrm{hpf}$ embryos. $\boldsymbol{D}, \boldsymbol{E}$, Expression pattern of olfm $1 a \mathrm{mRNA}$ in the head of a tail-bud stage embryo. Antisense probe for whole-mount in situ hybridization recognized olfm1a TR2 transcripts. Scale bar, $0.05 \mathrm{~mm}$. 
of the N1-293 truncated construct (Fig. $2 F$ ). The extended projection field of the optic nerve terminals in the optic tectum could be attributed to the acceleration of optic nerve growth, an increase in the branch number of the optic nerve axons, or facilitated arborization. The fact that truncated forms of Olfm 1 are more effective in the optic nerve implies that the $\mathrm{N}$-terminal part of Olfm 1 has a role to regulate the optic nerve extension different from the eye size regulations.

\section{Knockdown of zebrafish Olfm 1 synthesis by morpholino oligonucleotide injections}

To examine effects of inhibition of Olfm 1 synthesis during development, we used antisense MO injection. In a previous study, we demonstrated that $B M Z$ and $B M Y$ transcripts of the olfm $1 a$ and olfm $1 b$ genes (TR2 and TR4 forms, respectively) were detected by in situ hybridization starting from $16 \mathrm{hpf}$, whereas $A M Z$ and $A M Y$ transcripts of both genes (TR1 and TR3 forms) were detected later (Nakaya and Tomarev, 2007). We used a Q-PCR analysis to identify olfm 1 variants that were the most abundant at earlier developmental stages. Expression of the TR2 forms (the olfmla gene) was detected at the tail-bud stage of development (12 hpf), whereas the appearance of TR4 (the olfm $1 b$ gene) was delayed by $\sim 4 \mathrm{~h}$ until the 5 -somites stage (16 hpf) (Fig. 3A). Relative amounts of the long $(A M Z$ and $B M Z)$ and short ( $A M Y$ and $B M Y$ ) forms of olfmla and olfm $1 b$ mRNAs were also estimated by the Q-PCR using forward primers and reverse primers located in exon $4^{\prime}$ or 4 (Fig. $3 B, C)$. These experiments demonstrated that the long forms were preferentially transcribed from the olfm $1 a$ gene in early development ( $16 \mathrm{hpf}$ ). Conversely, both longer and shorter forms were transcribed from the olfm $1 b$ gene from $16 \mathrm{hpf}$ and onward. In summary, the $B M Z$ transcript of olfm 1 a predominate at early developmental stages. The earliest expression of olfmla was detected in the ventral region of the future head at the tail-bud stage (Fig. $3 D, E$ ).

To see the effect of simultaneous suppression of multiple Olfm1 isoforms, we injected Olfm1-MO, which was complementary to a stretch of identical nucleotides in the region encoding the initiator methionine in TR2 and TR4 transcripts. Efficiency of inhibition was tested in control experiments by injection of $5 \mathrm{ng}$ of this MO together with mouse Olfm1 RNA containing the zebrafish sequence complimentary to Olfm1MO. The Olfm1-MO completely inhibited detectable Olfm1 protein expression in the tail-bud stage embryos. A control MO and a mismatch MO differing from Olfm1-MO by five nucleotide replacements did not inhibit Olfm 1 (Fig. $1 C$, right). Analysis of morphological changes of the developing embryos demonstrated that $1.25-5 \mathrm{ng}$ of control MO did not produce pronounced morphological defects, whereas increasing concentrations of Olfm1-MO produced dose-dependent, damaging effects. When 5 ng of Olfm 1-MO was injected, the first detectable morphological changes were detected at $20 \mathrm{hpf}$. These included flattening of the head and the delay of eye development (data not shown). At $24 \mathrm{hpf}$, morphological defects included some cloudiness of the brain, indicating its degeneration, defective eye development, distorted body axis, the heart expansion, the rough surface of the skin, and cell dissociations from the dorsal surface of the body (data not shown). At later developmental stages (later than $35 \mathrm{hpf}$ ), Olfml-MO-injected embryos demonstrated reduced pigmentation of the eye and the body, smaller eyes, and shorter body length compared with embryos injected with control MO (Figs. $4 A-D, 5 A, B$ ). Analysis of sections through the cranial part of $72 \mathrm{hpf} \mathrm{MO}$-injected embryos revealed a hypoplastic tectum with strongly damaged marginal zone and the loss of mandibular and hyosymplectic cartilages (Fig. 4C,D).

Sectioning of the embryos demonstrated that the size of the retina was dramatically reduced, and all retinal layers were affected. The RGC layer was disorganized and contained fewer cells than in control retina. The inner nuclear layer contained degenerating cells, which were not observed in the control (Fig. 5C,D). Inner plexiform and outer nuclear layers appeared to be thinner 


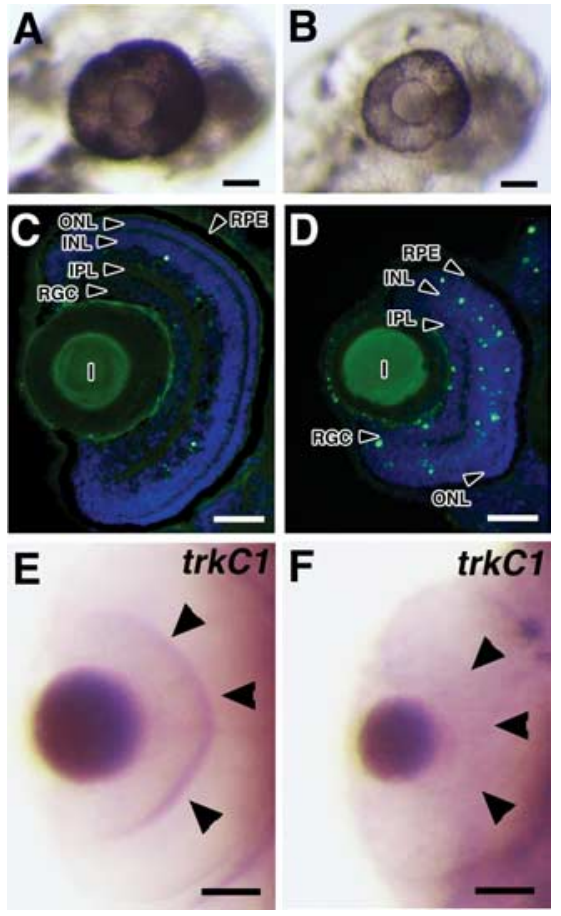

Figure 5. Effects of Olfm1-M0 injections on the eyes of $72 \mathrm{hpf}$ embryos. $A, B$, Embryos were injected with $5 \mathrm{ng}$ of control $(\boldsymbol{A})$ or Olfm1-M0 $(\boldsymbol{B})$. Olfm1-M0-injected embryos showed a reduction of eye size and pigmentation compared with embryos injected with control M0. C, D, TUNEL staining of eye sections from embryos injected with $5 \mathrm{ng}$ of control $\mathrm{MO}$ (C) or Olfm1-MO (D). $\boldsymbol{E}, \boldsymbol{F}$, In 3 days postfertilization embryos, $01 \mathrm{fm} 1-\mathrm{MO}(\boldsymbol{F})$ inhibited trkc1 expression in both $\mathrm{RGC}$ and inner plexiform layers compared with control MO (E). Scale bar, $0.05 \mathrm{~mm}$. IPL, Inner plexiform layer; INL, inner nuclear layer; $\mathrm{OPL}$, outer plexiform layer; $\mathrm{ONL}$, outer nuclear layer; $\mathrm{RPE}$, retinal pigmented epithelium; $\mathrm{RGC}$, retinal ganglion cells.

in Olfm1-MO-injected retinas. Pigmentation of the retinal pigment epithelium was reduced in Olfm1-MO-injected embryos. Terminal deoxynucleotidyl transferase-mediated biotinylated UTP nick end labeling (TUNEL) staining of the eye sections showed that there were more apoptotic nuclei in the RGC and inner nuclear cell layers of Olfm1-MO-treated $72 \mathrm{hpf}$ embryos than control embryos (Fig. 5C,D), indicating that the apoptosis might play an essential role in the reduction of the retina size in Olfm1-MO-injected embryos. Among genes tested, Olfm1-MO injection dramatically reduced the expression of a gene encoding tyrosine receptor kinase $\mathrm{C} 1$ ( $\operatorname{trkC1})$ in the RGCs and the inner plexiform layer (Fig. $5 E, F$ ), without affecting its expression in other parts of the embryo (data not shown). Similarly, immunostaining of the eye sections with antibodies specific for different retinal cell subtypes demonstrated (Fig. 6) substantial reduction of staining in the RGC ( $\mathrm{Hu}-\mathrm{C} / \mathrm{D}$ and $\mathrm{Zn}-5$ positive) and inner nuclear (Hu-C/D and PKC $\beta 1$-positive) layers, whereas staining in the outer nuclear layer were less affected (Zpr-1 positive). Staining of neuronal fibers in the optic nerve fiber layer (Zn-5) and bipolar cells (PKC $\beta 1)$ was reduced more dramatically than staining of the cell bodies with DAPI.

As a consequence of disorganization and reduction of cell number in the RGC layer, the optic nerve contained fewer nerve fibers, and these were not so densely packed (fasciculated) as in control eyes. These damaged fibers appeared less efficient in the transport of DiI to the optic tectum, the main target of the RGC axons, as was demonstrated by DiI injection (Fig. $7 A, B$ ). A reduction of the optic tectum size in Olfm1-MO-injected embryos also may contribute to the observed defects in arborization of the
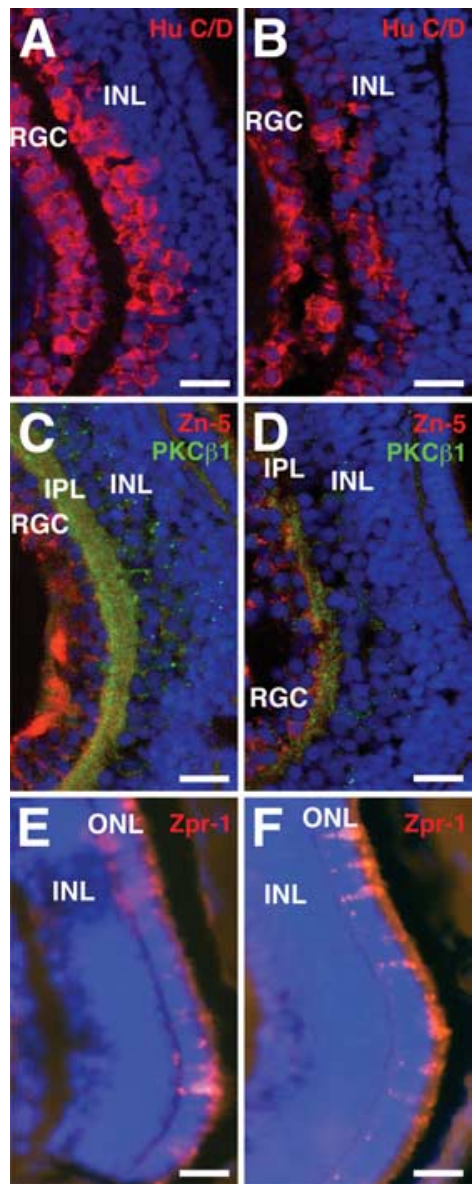

Figure 6. Effects of Olfm1-M0 injection on the expression of retinal marker proteins. Control M0 $(\boldsymbol{A}, \boldsymbol{C}, \boldsymbol{E})$ or Olfm1-MO $(\boldsymbol{B}, \boldsymbol{D}, \boldsymbol{F})$-injected embryos were fixed at $72 \mathrm{hpf}$, and the frozen sections at the eye level were prepared. The sections were stained for $\mathrm{Hu}-\mathrm{C} / \mathrm{D}(\boldsymbol{A}, \boldsymbol{B}$, red), $\mathrm{PKC} \beta 1$ ( $\boldsymbol{C}, \boldsymbol{D}$, green), Zn-5 (C, D, red), and Zpr-1 ( $\boldsymbol{E}, \boldsymbol{F}$, red) together with DAPI (blue). Scale bar, 0.05 $\mathrm{mm}$. IPL, Inner plexiform layer; INL, inner nuclear layer; $0 \mathrm{NL}$, outer nuclear layer; RGC, retinal ganglion cells.

optic nerve axon to the tectum. Olfm1-MO injection also reduced the axonal network in the spinal cord and the lateral line nerve extension as demonstrated by the staining of neuronal axons with antibodies against acetylated tubulin (Fig. 7C,D). Sites of the most pronounced damage, the eye and brain, approximately corresponded to sites of Olfm 1 expression at later developmental stages (72 hpf). olfm $1 a$ and olfm $1 b$ showed similar expression patterns, with the olf $m 1 b$ producing the strongest signals (Fig. $4 E$ ) (data not shown) and expressed in the RGC layer, inner plexiform layer, and optic nerve head. Expression was also detected in midbrain, especially in the optic tectum. Areas enriched in neuronal fibers such as the post-optic commissure and optic nerve stalk were also positive, suggesting the olf $m 1 \mathrm{mRNAs}$ are transported from cell bodies into these axons. Confocal immunofluorescence revealed that the Olfm 1 protein was present in the same place where the mRNA localized. The Olfm 1 antibodyspecific immunofluorescent signals were detected in the entire forebrain, with especially strong signals in the upper and peripheral optic tectum (Fig. $4 F$ ). In the eye, the protein was localized mainly in the RGCs and inner nuclear layer cells (Fig. 4 H). Olfm 1 showed cytoplasmic and probably extracellular localization and was excluded from nuclei. Staining without the primary antibody did not show any positive signal (Fig. 4G,I).

Effects of Olfm1-MO appeared to be specific because the in- 


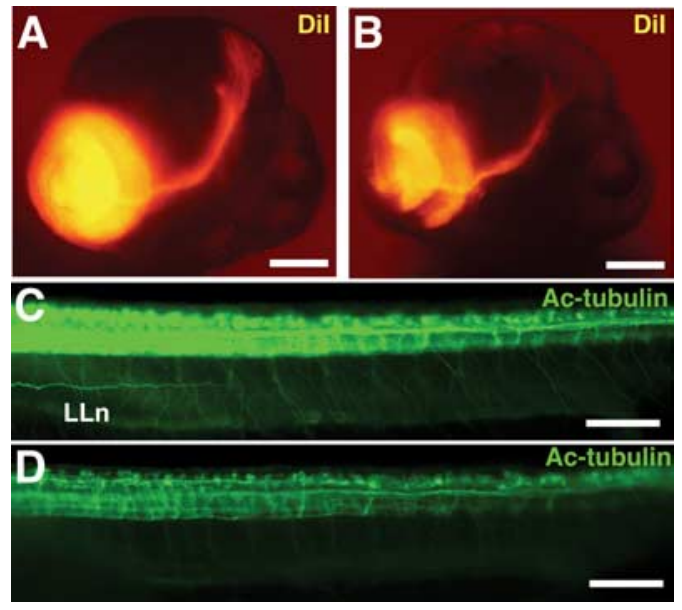

Figure 7. $\quad$ Olfm 1 is required for axonal extensions. $\boldsymbol{A}, \boldsymbol{B}$, Dil-labeled optic nerve of $80 \mathrm{hpf}$ control $(\boldsymbol{A})$ and $01 \mathrm{fm} 1-\mathrm{M} 0$-injected embryos $(\boldsymbol{B})$. A total of $1.25 \mathrm{ng}$ of $0 \mathrm{lfm} 1-\mathrm{M} 0$ produced inhibition of axon growth to the optic tectum. $\boldsymbol{C}, \boldsymbol{D}$, Control (C) and 0lfm1-M0-treated (D) 35 hpf embryos were stained with antibodies against acetylated tubulin. Note strong inhibition of neurons and their networks in 0lfm1-M0-injected embryos. LLn, Lateral line nerve. Scale bar, $0.1 \mathrm{~mm}$.

hibitory effect of MO on the eye size as well as defects to the body and brain were partially rescued by coinjection of RNA encoding the N1-152 Olfm 1 construct (Table 1). We tried several factors to rescue Olfm1-MO effects on the eye size. Among injected RNA tested, runxb RNA but not runxa RNA produced a significant rescue effect (data not shown). Zebrafish runx genes encode runtrelated transcription factors that are, similar to the olf $m 1$ genes, strongly expressed in sensory neuronal tissues such as trigeminal ganglia and Rohon-Beard neurons. However, runx genes have not been reported to be expressed in the eye (Kataoka et al., 2000; Burns et al., 2002). Injection of runxb RNA changed the levels of olfm $1 \mathrm{mRNAs}$ : expression of the olfm $1 \mathrm{a}$ gene at $24 \mathrm{hpf}$ increased $(154 \pm 12 \%$ for TR2 and $312 \pm 26 \%$ for TR 1$)$, whereas expression of the olfm $1 b$ gene did not change or decreased (118 $\pm 24 \%$ for TR3 and $74 \pm 21 \%$ for TR4). This suggests that runxb acts upstream of olf $\mathrm{m} 1$ and may regulate the olf $\mathrm{m} 1$ gene expression. In conclusion, these data support the idea that Olfm1 has an important developmental role in the differentiation of neural retina and may affect the axon growth.

\section{The effect of Olfm 1 on the expression of selected genes involved in eye development}

Because experimental changes in Olfm 1 expression alter eye size, we checked whether the expression domains of early eye field markers in the anterior neural plate are also affected. The gene expression patterns of $r \times 3$, which is the earliest eye field marker, and six3, which acts downstream of $r \times 3$, were analyzed by in situ hybridization. Similar to published results (Chuang et al., 1999), the $r \times 3$ expression was detected in the lateral optic primordia and ventral medial diencephalon from 12 to $15 \mathrm{hpf}$ embryos (Fig. $8 A$ ). Injection of Olfm1-MO reduced the lateral separation of the optic primordia expressing $r \times 3$ and increased the anteroposterior length of $r x 3$ expression (Fig. $8 B$ ). Coinjection of $z$ Olfm 1 RNA together with Olfm $1-\mathrm{MO}$ restored the $r \times 3$ expression pattern and made it similar to that observed in control embryos (Fig. 8C). This indicates that the effect of Olfm1-MO injection on $r \times 3$ expression is specific and attributable to reduction of Olfm 1 level. Expression of six 3 was dramatically extended in the anteroposterior direction after Olfm1-MO injection (Fig. 8E). The olfm1
RNA injection did not affect anteroposterior extent of six3 expression but increased the lateral separation of optic primordia (Fig. 8F).

Formation of the anteroposterior axis of the early forebrain is regulated by a number of genes, including wnt (Houart et al., 2002; Pézeron et al., 2006; Stigloher et al., 2006; Tendeng and Houart, 2006). It has been shown that injection of RNA encoding the Wnt inhibitor sFRP1 expands the area of six3 expression (Kim et al., 2007). Similarly, injection of RNA encoding another inhibitor of Wnt signaling WIF-1 leads to the anteroposterior expansion of $r \times 3$ expression comparable with the effect of Olfm 1-MO, although it did not show any obvious effect on the distance between both future eye fields (Fig. 9A,B). The effect of the wif-1 RNA injection on the $r \times 3$ expression was reversed by coinjection with olfm1 RNA. The anteroposterior expression of $r x 3$ returned to normal size in wif-1- and olfm1-injected embryos (Fig. 9C). wif-1 RNA injection not only expanded the expression of early eye markers but also enhanced the size of eyes at two developmental stages tested (Table 1). The increase in eye size by wif-1 was inhibited by a coinjection with olfm1 RNA but not with RNA encoding truncated forms of Olfm 1 . Together, these data indicate that WIF-1 and Olfm 1 may affect the same signaling pathways and that these proteins may interact with identical targets and/or possibly with each other.

\section{Interaction of Olfm 1 and WIF-1}

We used a TopFlash reporter system to check effects of WIF-1 and Olfm 1 on canonical Wnt signaling in zebrafish. A reporter plasmid, which contains luciferase preceded by the multimerized consensus sequence for the lymphocyte enhancer factor/T-cell factor, was injected into embryos, and the luciferase activity was measured in the lysates $24 \mathrm{~h}$ after injections (Fig. 9D). The basal level of luciferase activity attributable to endogenous wnts was markedly inhibited by the wif- 1 RNA injection, as expected. olfm 1 RNA alone did not have an effect on the luciferase activity. However, coinjection of olfm 1 RNA together with wif- 1 RNA reduced the inhibitory effect of wif-1, suggesting that there may be an antagonistic interaction between WIF-1 and Olfm1.

Possible physical interaction of Olfm 1 and WIF-1 was tested in cell culture experiments. NIH3T3 cells were cotransfected with cDNAs encoding Olfm 1 and WIF-1 or its WD domain fused to the IgG heavy chain. The WIF-1 WD domain is essential for interactions with Wnt proteins (Patthy, 2000). Because both Olfm 1 and WIF-1 are secreted proteins, coimmunoprecipitation was performed from conditioned medium of transfected cells. The results demonstrated (Fig. 10) that Olfm1 interacts with WIF-1 and that the WD domain is sufficient for this interaction. We tested the truncated forms of Olfm 1 for the interaction. Interestingly, the Flag-tagged truncated Olfm1 are much less secreted in cell culture, and, because of the amount of protein in the culture medium, they did not show a detectable interaction with the WIF-1 compared with the full-length form. Although the immunoprecipitation was also performed using in vivo tissue, it did not give detectable results because of the inability of antiOlfm 1 and anti-WIF-1 antibodies to detect the endogenous intact protein. It must be confirmed by producing other antibodies in future.

\section{Discussion}

Zebrafish is a convenient system to analyze functions of genes and proteins in development. Olfactomedin domain-containing proteins play important roles in normal development and disease. There are two olfm 1 genes in zebrafish, and, in this work, we 
Table 1. The eye size regulation by olfm 1 and wif-1

\begin{tabular}{llll}
\hline & & Size of eyes $(\mathrm{mm})$ & $4.5 \mathrm{dpf}$ \\
\cline { 3 - 4 } & Dose $(\mathrm{ng})$ & $2.5 \mathrm{dpf}$ & \\
\hline Olfm1-M0 & & $0.274 \pm 0.002$ & \\
EGFP + 0lfm1-M0 & 5 & $0.185 \pm 0.003^{* *}$ & \\
Olfm1 + 0lfm1-M0 & $0.1+5$ & $0.191 \pm 0.003$ & \\
N1-293 + 0lfm1-M0 & $0.1+5$ & $0.202 \pm 0.010$ & $0.361 \pm 0.002$ \\
N1-152+ 0lfm1-M0 & $0.1+5$ & $0.199 \pm 0.005$ & $0.366 \pm 0.002$ \\
& $0.1+5$ & $0.206 \pm 0.005^{*, \neq}$ & $0.363 \pm 0.002$ \\
WIF-1 & & $0.309 \pm 0.002$ & $0.375 \pm 0.002^{* *}$ \\
WIF-1 & 0.01 & $0.316 \pm 0.002^{*}$ & $0.373 \pm 0.003^{* *}$ \\
WIF-1 & 0.03 & $0.319 \pm 0.003^{* *}$ & \\
WIF-1 & 0.05 & $0.323 \pm 0.003^{* *}$ & \\
EGFP & 0.10 & $0.327 \pm 0.003^{* *}$ & \\
WIF-1 & & $0.316 \pm 0.002$ & \\
WIF-1 + EGFP & 0.05 & $0.305 \pm 0.003$ & \\
WIF-1 + 0lfm1 & 0.05 & $0.327 \pm 0.004^{*}$ & \\
WIF-1 + N1-293 & $0.05+0.05$ & $0.328 \pm 0.002$ & \\
WIF-1 + N1-152 & $0.05+0.05$ & $0.312 \pm 0.004^{*, \dagger}$ & \\
\hline
\end{tabular}

${ }^{*} p<0.05,{ }^{* *} p<0.01 .{ }^{\dagger}$ Against WIF-1-injected embryos. ${ }^{\ddagger}$ Against Olfm1-M0-injected embryos. dpf, Days postfertilization.
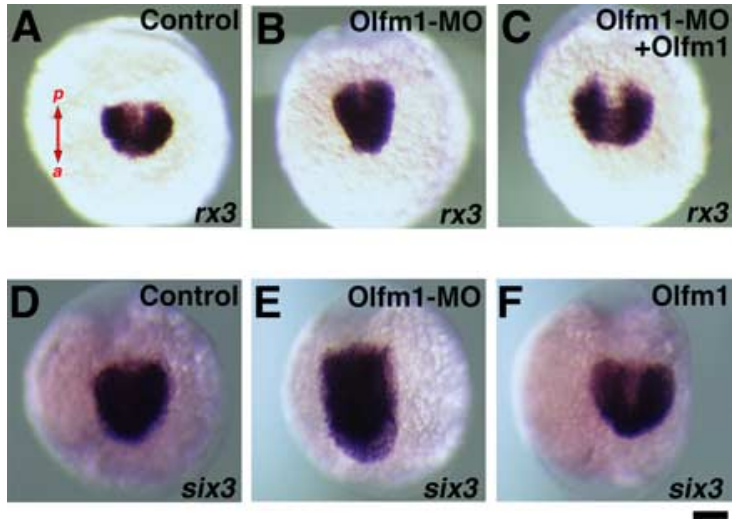

Figure 8. Changes in the expression patterns of early eye markers after olfm1 RNA or Olfm1-M0 injection. Embryos were injected with $0.1 \mathrm{ng}$ of $z 0 / \mathrm{fm} 1 \mathrm{RNA}(\boldsymbol{F}), 5 \mathrm{ng}$ of Olfm1-M0, and $0.1 \mathrm{ng}$ of EGFPRNA $(\boldsymbol{B}, \boldsymbol{E})$ or a mixture of $201 \mathrm{fm} 1 \mathrm{RNA}$ and 0 lfm1-MO $(\boldsymbol{C})$ and were analyzed for rx3 $(\boldsymbol{A}-\boldsymbol{C})$, and six3 $(\boldsymbol{D}-\boldsymbol{F})$ mRNA expression at $13 \mathrm{hpf}$. Control embryos $(\boldsymbol{A}, \boldsymbol{D})$ were injected with $0.1 \mathrm{ng}$ of EGFP RNA. Shown are dorsal views of the future heads. a, Anterior; $\mathrm{p}$, posterior. Scale bar, $0.1 \mathrm{~mm}$

studied the effects of their inhibition by MOs and overexpression by injection of Olfm RNAs. Overexpression of the AMZ form but not the BMY form of Olfm 1 led to mild enlargement of the retina. It was reported previously that injection of RNA encoding the AMY form of olfm 1 into Xenopus embryos induced expansion of neural territories, especially in the retina (Moreno and BronnerFraser, 2005). Conversely, a decrease in Olfm1 levels by MO led to a reduction in eye size, inhibition of optic nerve extension, and reduced pigmentation of the eye. Reduction in eye size has also been reported after inhibition of Olfm2, another olfactomedin domain-containing protein, by a MO injection (Lee et al., 2008). Other changes observed after the Olfm1-MO injection into zebrafish included defects in the brain and the mandibular and the hyosymplectic cartilages, reduced body pigmentation, and distorted body axis. It is interesting to note that the injection of Olfm2-MO also produced defects in cartilaginous structures, but, in this case, frontal arches (mandibular and hyoid) were less affected than caudal branchial arches (Lee et al., 2008). Most of Olfm1-MO effects were partially rescued by the coinjection of the N1-152 Olfm 1 construct, whereas other constructs were less effective. We could not achieve a complete rescue even by the injection of higher amount of the olfm1 RNA. Similarly, the damaging effects of Olfm2-MO injections could not be restored by injection of olfm2 RNA (Lee et al., 2008). Some defects caused by the Olfm1-MO injection could be predicted on the basis of previous observations in Xenopus and chicken (Barembaum et al., 2000; Sakuragi et al., 2006). For example, the reduced pigmentation and the loss of cranial cartilages caused by the Olfm1-MO injection indicate its possible effects on neural crest cell differentiation into melanocytes and cranial cartilages. Brain and eye defects may result from the involvement of Olfm 1 in neurogenesis. At the same time, it should be mentioned that the elimination of the central M part from all isoforms of Olfm1 in mice did not produce visible phenotype (Cheng et al., 2007). One possible explanation for this observation is that the elimination of the M part from all Olfm 1 forms does not prevent the formation of alternatively spliced forms of Olfm 1 that were lacking the M part but contained the olfactomedin domain. These products may perform some functions of full-length Olfm1. Our preliminary experiments also indicate that, in rodents, Olfm 2 shows an expression pattern very similar to that for $\mathrm{Olfm} 1$ and may produce a compensatory effect (V. V. Senatorov and S. I. Tomarev, unpublished observations). This is also true for zebrafish olfm 1 and olfm2 genes that show overlapping but not identical expression patterns (Nakaya and Tomarev, 2007; Lee et al., 2008).

A possible explanation for the observed eye defects may be the change in the pattern of expression of $r \times 3$ and six3. These genes are essential for early eye development. Domains of their expression in the optic primordia were more laterally separated by the olfm1 RNA injection and narrowed by the Olfm1-MO injection. A change in the lateral separation of the eye primordia was also observed for the $r x 2$ gene expression (data not shown). This effect can be caused by changes in sonic hedgehog ( $\mathrm{SHH}$ ) expression, which is produced by the notochord and floor plate in the midline and is involved in the separation of the eye fields (Ekker et al., 1995; Martí et al., 1995; Shimamura et al., 1995; Li et al., 1997; Rorick et al., 2007), although Olfm2-MO did not change the pattern of SHH expression (Lee et al., 2008).

Most probably, Olfm1, similar to some other secreted glycoproteins, interacts with cellular receptor(s) and stimulates or inhibits intracellular signaling pathways. It has been reported that gliomedin interacts specifically with neurofascin and neuronglia-related cell adhesion molecule (Eshed et al., 2005). hGC-1, also known as GW112, OLFM4, and hOlfD, may interact with cell surface lectins and cadherins (Liu et al., 2006). Finally, amassin, an extracellular glycosylated protein found in the coelomic fluid of sea urchin, may bind to an unidentified cell surface protein (Hillier and Vacquier, 2003). Recent data indicate that different forms of Olfm 1 may interact with $\beta$-dystrobrevin (Veroni et al., 2007). $\beta$-Dystrobrevin is a component of the dystrophinassociated protein complex that may link the actin cytoskeleton to the extracellular matrix and may serve as a scaffold for signaling proteins.

We found that Olfm1 may interact with WIF-1, a secreted inhibitor of Wnt signaling. WIF-1 is highly expressed in mam- 
malian retina (Hunter et al., 2004). In the embryonic mouse retina (embryonic day 16 ), it is expressed in the RGCs and the inner nuclear layer, whereas in the adult retina, it is more highly expressed in the inner and outer nuclear layers (Hunter et al., 2004). Therefore, WIF-1 and Olfm 1 show overlapping expression patterns in the developing and adult eyes. Wnt signaling is one of the major regulatory cascades important for normal embryogenesis of multicellular organisms. It has been demonstrated that the interaction of Wnt and its antagonists plays a critical role at early stages of eye development (Houart et al., 2002; Zilinski et al., 2004; Pézeron et al., 2006; Stigloher et al., 2006; Tendeng and Houart, 2006). Wnts produced at the hindbrain work as a posteriorizing signal toward the forebrain, whereas its inhibitors counteract to Wnt action and stimulate the anteriorization to establish the forebrain and future eye area. Our study indicates that Olfm 1 may modulate Wnt signaling by binding the Wnt inhibitors and Wnt receptors. Identification of Wnt receptors interacting with Olfm1 is currently in progress.

One of the key observations of this paper concerns possible functions of Olfm 1 . Injection of RNA encoding the olfactomedin domain-lacking isoforms or long (BMZ) form of Olfm1, which was a predominant transcript in the course of early zebrafish development (Fig. 3), increased the thickness of the optic nerve and produced more extended projection field in the tectum in 72 hpf embryos (Fig. 2). The olfactomedin domain-lacking isoforms produced more pronounced effects than full-length Olfm 1 . It has been reported previously that short and long forms of Olfm 1 may have different functional roles (Moreno and Bronner-Fraser, 2005). Although the Olfm1-MO injection inhibited the neurite extension from the motor and RohonBeard neurons, the injection of olfm1 RNA did not lead to detectable changes in the axon network produced by these neurons, as judged by staining with antibodies against acetylated tubulin (data not shown). At present, we do not fully understand why the truncated forms of olfactomedin are more effective in increasing thickness of the optic nerve than their full-length counterparts. Available data suggest that truncated and full-length forms of olfactomedin may interact with different proteins. For example, the BMY but not BMZ form of olfactomedin interacts with WAVE1 (Cheng et al., 2007), an actin regulating protein that is present along the leading edge of the neuronal growth cones. It is possible that such interaction promote the optic nerve growth. Another possibility is that short and long forms of olfactomedin are secreted with different efficiencies and therefore may exercise different effects on the optic nerve growth via interactions with presumptive receptors and extracellular matrix. Injections of RNAs encoding short forms of Olfm 1 may also shift the balance between short and long forms compared with injection of the long forms. It is feasible to suggest that Olfm1, by modulating
Wnt signaling pathways, may change the activity of several factors, which were shown to be involved in the axon branching or it may affect interactions of the growing axons with the supporting glia cells. Our preliminary results indicate that Olfm 1 may induce neurite outgrowth in different experimental systems, including nerve growth factor-stimulated PC12 cells (H.-S.L., N.N., and S.I.T, unpublished observations) and embryonic rat hippocampal neurons (N.N. and S.I.T, unpublished observations). Altogether, these data indicate that Olfm 1 has an essential role in axon extension. Available data suggest that Olfm 1 may be essential for neural development and neural crest generation (Barembaum et al., 2000; Moreno and Bronner-Fraser, 2001). One of the forms of Olfm1, BMY (also known as pancortin-2), may serve as a mediator of ischemia-induced apoptosis of neurons in the adult cerebral cortex (Cheng et al., 2007). Recent data suggest that olfactomedin-2 is an important regulator of development of the anterior nervous system (Lee et al., 2008). Other olfactomedin domain-containing proteins, for example, Xenopus tiarin, may participate in the specification of the dorsal neural tube (Tsuda et al., 2002). Gliomedin appears to mediate Schwann cell-axon interaction and molecular assembly of the nodes of Ranvier (Eshed et al., 2005). Sea urchin amassin mediates the massive intercellu- 


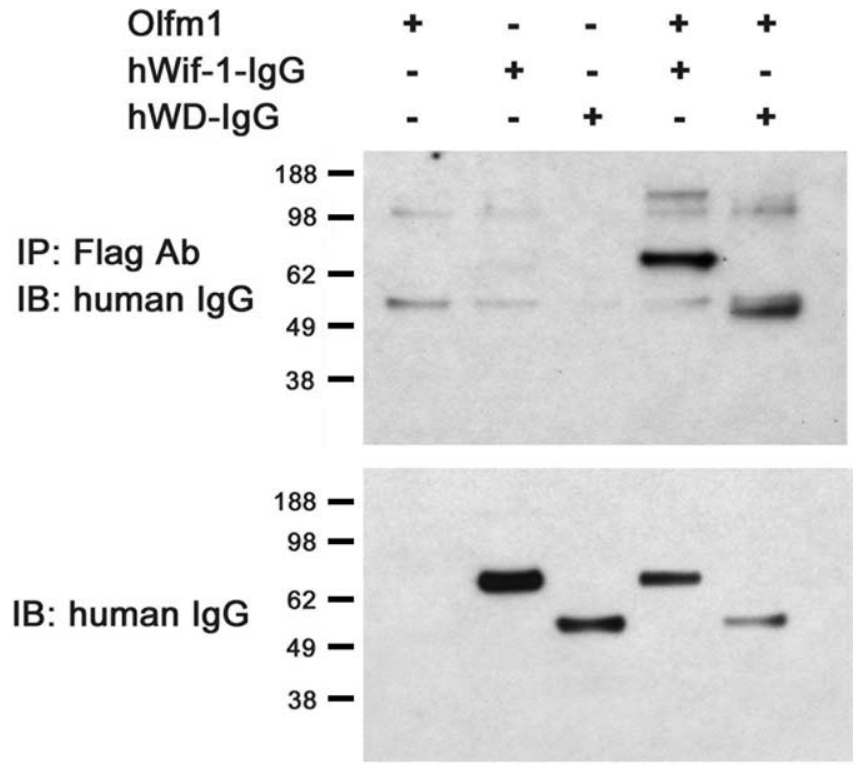

Figure 10. Physical interaction of Olfm 1 and WIF-1. HEK-293 cells were cotransfected with indicated constructs. Conditioned medium was collected $48 \mathrm{~h}$ after transfection, and protein complexes were precipitated with Flag antibody beads. Proteins eluted from the beads were separated by SDS-PAGE and probed with peroxidase-conjugated antibodies against human IgG (top). Bottom shows Western blot of conditioned medium before immunoprecipitation probed with peroxidase-conjugated antibodies against human lgG.

lar adhesion of coelomocytes, primitive immune cells found in echinoderms (Hillier and Vacquier, 2003).

In summary, our data demonstrate that the olfm 1 genes are essential for development of several organs, including eye and brain. In the eye, Olfm 1 may be essential for the early eye determination, RGC differentiation, and the optic nerve axons growth and targeting of the optic tectum. Although mutations in the olfm1 gene have not been found yet, mutations in two other family members, myocilin and olfm2, appear to be contributing factors to human glaucoma (Stone et al., 1997; Funayama et al., 2006). Expression of olfm 1 in RGCs and in the optic nerve makes olfm1 a good candidate gene for mutations that might also contribute to human glaucoma.

\section{References}

Barembaum M, Moreno TA, LaBonne C, Sechrist J, Bronner-Fraser M (2000) Noelin-1 is a secreted glycoprotein involved in generation of the neural crest. Nat Cell Biol 2:219-225.

Burns CE, DeBlasio T, Zhou Y, Zhang J, Zon L, Nimer SD (2002) Isolation and characterization of runxa and runxb, zebrafish members of the runt family of transcriptional regulators. Exp Hematol 30:1381-1389.

Cheng A, Arumugam TV, Liu D, Khatri RG, Mustafa K, Kwak S, Ling HP, Gonzales C, Xin O, Jo DG, Guo Z, Mark RJ, Mattson MP (2007) Pancortin-2 interacts with WAVE1 and Bcl-xL in a mitochondriaassociated protein complex that mediates ischemic neuronal death. J Neurosci 27:1519-1528.

Chuang JC, Mathers PH, Raymond PA (1999) Expression of three Rx homeobox genes in embryonic and adult zebrafish. Mech Dev 84:195-198.

Danielson PE, Forss-Petter S, Battenberg EL, deLecea L, Bloom FE, Sutcliffe JG (1994) Four structurally distinct neuron-specific olfactomedinrelated glycoproteins produced by differential promoter utilization and alternative mRNA splicing from a single gene. J Neurosci Res 38:468-478.

Ekker SC, McGrew LL, Lai CJ, Lee JJ, von Kessler DP, Moon RT, Beachy PA (1995) Distinct expression and shared activities of members of the hedgehog gene family of Xenopus laevis. Development 121:2337-2347.

Eshed Y, Feinberg K, Poliak S, Sabanay H, Sarig-Nadir O, Spiegel I, Bermingham JR, Jr, Peles E (2005) Gliomedin mediates Schwann cell-axon interaction and the molecular assembly of the nodes of Ranvier. Neuron $47: 215-229$.
Funayama T, Mashima Y, Ohtake Y, Ishikawa K, Fuse N, Yasuda N, Fukuchi T, Murakami A, Hotta Y, Shimada N (2006) SNPs and interaction analyses of noelin 2, myocilin, and optineurin genes in Japanese patients with open-angle glaucoma. Invest Ophthalmol Vis Sci 47:5368-5375.

Hemish J, Nakaya N, Mittal V, Enikolopov G (2003) Nitric oxide activates diverse signaling pathways to regulate gene expression. J Biol Chem 278:42321-42329.

Hillier BJ, Vacquier VD (2003) Amassin, an olfactomedin protein, mediates the massive intercellular adhesion of sea urchin coelomocytes. J Cell Biol 160:597-604

Houart C, Caneparo L, Heisenberg C, Barth K, Take-Uchi M, Wilson S (2002) Establishment of the telencephalon during gastrulation by local antagonism of Wnt signaling. Neuron 35:255-265.

Hsieh JC, Kodjabachian L, Rebbert ML, Rattner A, Smallwood PM, Samos CH, Nusse R, Dawid IB, Nathans J (1999) A new secreted protein that binds to Wnt proteins and inhibits their activities. Nature 398:431-436.

Hunter DD, Zhang M, Ferguson JW, Koch M, Brunken WJ (2004) The extracellular matrix component WIF-1 is expressed during, and can modulate, retinal development. Mol Cell Neurosci 27:477-488.

Joe MK, Sohn S, Hur W, Moon Y, Choi YR, Kee C (2003) Accumulation of mutant myocilins in ER leads to ER stress and potential cytotoxicity in human trabecular meshwork cells. Biochem Biophys Res Commun 312:592-600.

Kataoka H, Ochi M, Enomoto K, Yamaguchi A (2000) Cloning and embryonic expression patterns of the zebrafish Runt domain genes, runxa and runxb. Mech Dev 98:139-143.

Kim HS, Shin J, Kim SH, Chun HS, Kim JD, Kim YS, Kim MJ, Rhee M, Yeo SY, Huh TL (2007) Eye field requires the function of Sfrp1 as a Wnt antagonist. Neurosci Lett 414:26-29.

Lee JA, Anholt RR, Cole GJ (2008) Olfactomedin-2 mediates development of the anterior central nervous system and head structures in zebrafish. Mech Dev 125:167-181.

Li H, Tierney C, Wen L, Wu JY, Rao Y (1997) A single morphogenetic field gives rise to two retina primordia under the influence of the prechordal plate. Development 124:603-615.

Liu W, Chen L, Zhu J, Rodgers GP (2006) The glycoprotein hGC-1 binds to cadherin and lectins. Exp Cell Res 312:1785-1797.

Liu Y, Vollrath D (2004) Reversal of mutant myocilin non-secretion and cell killing: implications for glaucoma. Hum Mol Genet 13:1193-1204.

Martí E, Takada R, Bumcrot DA, Sasaki H, McMahon AP (1995) Distribution of Sonic hedgehog peptides in the developing chick and mouse embryo. Development 121:2537-2547.

Moreno TA, Bronner-Fraser M (2001) The secreted glycoprotein Noelin-1 promotes neurogenesis in Xenopus. Dev Biol 240:340-360.

Moreno TA, Bronner-Fraser M (2002) Neural expression of mouse Noelin1/2 and comparison with other vertebrates. Mech Dev 119:121-125.

Moreno TA, Bronner-Fraser M (2005) Noelins modulate the timing of neuronal differentiation during development. Dev Biol 288:434-447.

Nagano T, Nakamura A, Mori Y, Maeda M, Takami T, Shiosaka S, Takagi H, Sato M (1998) Differentially expressed olfactomedin-related glycoproteins (Pancortins) in the brain. Brain Res Mol Brain Res 53:13-23.

Nakaya N, Tomarev S (2007) Expression patterns of alternative transcripts of the zebrafish olfactomedin 1 genes. Gene Expr Patterns 7:723-729.

Patthy L (2000) The WIF module. Trends Biochem Sci 25:12-13.

Pézeron G, Anselme I, Laplante M, Ellingsen S, Becker TS, Rosa FM, Charnay P, Schneider-Maunoury S, Mourrain P, Ghislain J (2006) Duplicate sfrp1 genes in zebrafish: sfrpla is dynamically expressed in the developing central nervous system, gut and lateral line. Gene Expr Patterns 6:835-842.

Rorick AM, Mei W, Liette NL, Phiel C, El-Hodiri HM, Yang J (2007) PP2A: B56epsilon is required for eye induction and eye field separation. Dev Biol 302:477-493.

Sakuragi M, Sasai N, Ikeya M, Kawada M, Onai T, Katahira T, Nakamura H, Sasai Y (2006) Functional analysis of chick ONT1 reveals distinguishable activities among olfactomedin-related signaling factors. Mech Dev 123:114-123.

Shimamura K, Hartigan DJ, Martinez S, Puelles L, Rubenstein JL (1995) Longitudinal organization of the anterior neural plate and neural tube. Development 121:3923-3933.

Snyder DA, Rivers AM, Yokoe H, Menco BP, Anholt RR (1991) Olfactomedin: purification, characterization, and localization of a novel olfactory glycoprotein. Biochemistry 30:9143-9153. 
Stigloher C, Ninkovic J, Laplante M, Geling A, Tannhäuser B, Topp S, Kikuta H, Becker TS, Houart C, Bally-Cuif L (2006) Segregation of telencephalic and eye-field identities inside the zebrafish forebrain territory is controlled by Rx3. Development 133:2925-2935.

Stone EM, Fingert JH, Alward WL, Nguyen TD, Polansky JR, Sunden SL, Nishimura D, Clark AF, Nystuen A, Nichols BE, Mackey DA, Ritch R, Kalenak JW, Craven ER, Sheffield VC (1997) Identification of a gene that causes primary open angle glaucoma. Science 275:668-670.

Tendeng C, Houart C (2006) Cloning and embryonic expression of five distinct sfrp genes in the zebrafish Danio rerio. Gene Expr Patterns 6:761-771.

Torrado M, Trivedi R, Zinovieva R, Karavanova I, Tomarev SI (2002) Optimedin: a novel olfactomedin-related protein that interacts with myocilin. Hum Mol Genet 11:1291-1301.

Toyama R, O'Connell ML, Wright CV, Kuehn MR, Dawid IB (1995) Nodal induces ectopic goosecoid and lim1 expression and axis duplication in zebrafish. Development 121:383-391.

Tsuda H, Sasai N, Matsuo-Takasaki M, Sakuragi M, Murakami Y, Sasai Y (2002) Dorsalization of the neural tube by Xenopus tiarin, a novel patterning factor secreted by the flanking nonneural head ectoderm. Neuron 33:515-528.

Veroni C, Grasso M, Macchia G, Ramoni C, Ceccarini M, Petrucci TC, Ma- cioce P (2007) beta-dystrobrevin, a kinesin-binding receptor, interacts with the extracellular matrix components pancortins. J Neurosci Res 85:2631-2639.

Volynski KE, Silva JP, Lelianova VG, Atiqur Rahman M, Hopkins C, Ushkaryov YA (2004) Latrophilin fragments behave as independent proteins that associate and signal on binding of LTX(N4C). EMBO J 23:4423-4433.

Westerfield M (2000) The zebrafish book. A guide for the laboratory use of zebrafish (Danio rerio). Eugene, OR: University of Oregon Press.

Yoda H, Hirose Y, Yasuoka A, Sasado T, Morinaga C, Deguchi T, Henrich T, Iwanami N, Watanabe T, Osakada M, Kunimatsu S, Wittbrodt J, Suwa H, Niwa K, Okamoto Y, Yamanaka T, Kondoh H, Furutani-Seiki M (2004) Mutations affecting retinotectal axonal pathfinding in Medaka, Oryzias latipes. Mech Dev 121:715-728.

Zeng LC, Han ZG, Ma WJ (2005) Elucidation of subfamily segregation and intramolecular coevolution of the olfactomedin-like proteins by comprehensive phylogenetic analysis and gene expression pattern assessment. FEBS Lett 579:5443-5453.

Zilinski C, Brownell I, Hashimoto R, Medina-Martinez O, Swindell EC, Jamrich M (2004) Expression of FoxE4 and Rx visualizes the timing and dynamics of critical processes taking place during initial stages of vertebrate eye development. Dev Neurosci 26:294-307. 